\title{
The association between BRCA1 gene polymorphism and cancer risk: a meta-analysis
}

\author{
Gui-Ping $\mathrm{Xu}^{1,{ }^{*}}$, Qing Zhao ${ }^{2, *}$, Ding Wang ${ }^{2}$, Wen-Yue $\mathrm{Xie}^{3}$, Li-Jun Zhang ${ }^{2}$, Hua Zhou ${ }^{2}$, \\ Shi-Zhi Chen ${ }^{2}$ and Li-Fang Wu ${ }^{2}$ \\ ${ }^{1}$ Transfusion Department, The Second Affiliated Hospital of Chongqing Medical University, Chongqing, China \\ ${ }^{2}$ Department of Laboratory Medicine, The Second Affiliated Hospital of Chongqing Medical University, Chongqing, China \\ ${ }^{3}$ Department of Oncology, The Second Affiliated Hospital of Chongqing Medical University, Chongqing, China \\ "These authors contributed equally to this work
}

Correspondence to: Li-Fang Wu, email: lifangwu0408@hotmail.com

Keywords: BRCA1; polymorphism; meta-analysis; cancer

Received: January 07, $2017 \quad$ Accepted: November 17, $2017 \quad$ Published: January 06, 2018

Copyright: $\mathrm{Xu}$ et al. This is an open-access article distributed under the terms of the Creative Commons Attribution License 3.0 (CC BY 3.0), which permits unrestricted use, distribution, and reproduction in any medium, provided the original author and source are credited.

\section{ABSTRACT}

Many studies have reported that BRCA1 polymorphisms are associated with cancer risk, but the results remain controversial. The purpose of this meta-analysis is to evaluate the relationship between BRCA1 polymorphisms (rs799917, rs1799950, rs1799966, or rs16941) and cancer risk. Relevant studies were identified via a systematic search of the PubMed, Embase, and Web of Science databases up to July 31,2017 . Odds ratios (ORs) with $95 \%$ confidence intervals (CIs) were calculated to examine the strength of the associations. Thirty-five studies published in 19 publications involving 28,094 cases and 50,657 controls were included in this metaanalysis. There was no obvious association between rs799917, rs1799966, or rs16941 polymorphisms and overall cancer risk in any genetic models. However, subgroup analyses revealed that the rs799917 polymorphism could decrease the risk of cervical cancer, esophageal squamous cell carcinoma (ESCC), gastric cancer, and non-Hodgkin lymphoma (NHL) among Asian populations in one or more genetic models and that rs16941 could increase overall cancer risk among Caucasian populations in the homozygote and recessive models. Our meta-analysis also indicated that rs1799950 could decrease the breast cancer (BC) risk among Caucasian populations in the homozygote and recessive models. In summary, our results suggest that BRCA1 polymorphisms may play an important role in the etiology of cancer. However, due to the limited number of studies, these findings should be confirmed by new studies with larger sample sizes that address various types of cancer.

\section{INTRODUCTION}

Year by year, cancer incidence and mortality are increasing worldwide, seriously affecting human health and generating a huge economic burden. Cancer is thought to be the result of interactions between genes and environmental factors [1], such as tobacco use, alcohol intake, overweight and infection [2]. Single-nucleotide polymorphisms (SNPs) are the most common genetic alterations between individuals, and it is reported that SNPs in DNA repair genes have a relationship with a variety of cancers [3].
$B R C A 1$ is a tumor suppressor gene located on chromosome 17q21. It was mapped in 1990 and cloned in 1994 [4, 5]. The BRCA1 gene was first identified as a strong candidate gene influencing susceptibility to breast and ovarian cancer [5]. BRCA1 is comprised of multiple functional domains and interacts with many proteins, including tumor suppressors, oncogenes, DNA damage repair proteins, cell cycle regulators, and transcriptional activators and repressors $[6,7]$. BRCA1 deficiency can lead to defects in the $\mathrm{S}$ phase, G2/M phase, and spindle checkpoints, causing genetic instability and thus triggering DNA damage response, further increasing the risk of 
tumor formation [8-10]. It is reported that BRCA1 is expressed in many tissues, such as the lymph nodes, skin, bladder, cervix, liver, uterus, prostate, pancreas, lung, kidney, bone, brain, and is related to various types of cancer, including breast, ovarian, endometrial, pancreatic, prostate, and colon cancers [11-13].

Many studies have been conducted to examine the association between $B R C A 1$ polymorphisms and the risk of various cancers, including breast cancer, ovarian cancer (OC), cervical cancer, ESCC, gastric cancer, chronic myeloid leukemia (CML), and NHL [14-32]; however, the results remain inconsistent or even contradictory. For example, Dunning et al. found that rs799917 showed no significant association with breast cancer [14], while Nicoloso et al. suggested that rs799917 increased the risk of breast cancer [23]. Studies regarding rs1799966, rs1799950, and rs16941 are similarly inconsistent, so we performed a meta-analysis to more precisely determine the association between $B R C A 1$ polymorphisms and cancer risk.

\section{RESULTS}

\section{Characteristics of the studies}

The flow diagram of the study selection process is shown in Figure 1. A total of 102 articles were obtained from a database search and from other sources. After duplicate removal and subsequent title and abstract assessment, 26 articles were left for further full-text review. Based on the inclusion and exclusion criteria, 19 articles remained [14-32]. One article containing data regarding various types of cancer was treated as independent studies [14], and there were seven articles containing studies of various BRCA1 polymorphisms $[14,16,17,21,22,24,27]$. Altogether, 35 studies contained in 19 articles involving 28,094 cases and 50,657 controls were included in this meta-analysis. The main characteristics of the included studies are summarized in Table 1. These articles were published from 1997 to 2016. Among them, ten articles were carried out in Caucasian populations, six in Asian populations, and three in other populations. The quality of the studies was evaluated based on a quality assessment scale (Supplementary Table 1). The distributions of genotypes and allele frequencies in the cases and controls are shown in Table 2.

\section{Meta-analysis of rs799917}

We assessed the association between the BRCA1 rs799917 polymorphism and cancer risk by calculating the ORs and their 95\% CI using the following five genetic models: the allele model ( $\mathrm{T}$ vs. $\mathrm{C}$ ), the homozygote model (TT vs. CC), the heterozygote model (CT vs. CC), the dominant model $(\mathrm{TT}+\mathrm{CT}$ vs. $\mathrm{CC})$, and the recessive model (TT vs. CT $+\mathrm{CC}$ ). The main results of the meta- analysis for rs 799917 polymorphism are shown in Table 3 . In total, there were sixteen studies with 11,074 cases and 18,205 controls for rs799917 polymorphism. In the overall analysis, no significant association was observed between rs 799917 polymorphism and cancer risk in any genetic models (Table 3).

In the subgroup analyses by ethnicity, we observe a significant association between rs799917 polymorphism and cancer risk among Asian populations in the allele, homozygote, and recessive models (T vs. C: OR, 0.89, 95\% Cl, 0.80-0.99, $P=0.032$; TT vs. CC: OR, $0.72,95 \%$ $\mathrm{Cl}, 0.56-0.93, P=0.011$; TT vs. CT $+\mathrm{CC}: \mathrm{OR}, 0.72,95 \%$ $\mathrm{Cl}, 0.58-0.89, P=0.003)$.

In the subgroup analyses by cancer type, we found that there was no significant association between rs799917 polymorphism and breast cancer risk either in the overall population or in the Caucasian, Asian, or other populations (Table 3 and Figure 2), which is consistent with previous reports [33]. There were eight studies included in our meta-analysis regarding rs799917 polymorphism and the risk of non-breast cancer. Among these, four studies were carried out in Asian populations, and four studies carried out in Caucasian or other populations. The studies on Asians demonstrated that rs 799917 polymorphism could decrease the risk of cervical cancer, ESCC, gastric cancer, and NHL in one or more genetic models (for cervical cancer: TT vs. CC: OR, 0.64, 95\% Cl, 0.41-0.99, $P=$ 0.046; TT vs. CT + CC: OR, $0.63,95 \% \mathrm{Cl}, 0.41-0.95, P=$ 0.029; for ESCC: T vs. C: OR, 0.81, 95\% Cl, 0.72-0.92, $P=0.001$; TT vs. CC: OR, $0.59,95 \% \mathrm{Cl}, 0.45-0.77, P<$ $0.001 ;$ TT + CT vs. CC: OR, $0.84,95 \% \mathrm{Cl}, 0.71-1.00, P$ $=0.045$; TT vs. CT + CC: OR, 0.61, 95\% Cl, 0.48-0.78, $P<0.001$; for gastric cancer: T vs. C: OR, $0.76,95 \% \mathrm{Cl}$ , 0.65-0.88, $P<0.001$; TT vs. CC: OR, 0.48, 95\% Cl, $0.34-0.68, P<0.001$; TT + CT vs. CC: OR, $0.79,95 \%$ $\mathrm{Cl}, 0.64-0.98, P=0.030$; TT vs. CT $+\mathrm{CC}: \mathrm{OR}, 0.51,95 \%$ Cl, 0.37-0.71, $P<0.001$; for NHL: T vs. C: OR, 0.86, $95 \% \mathrm{Cl}, 0.75-0.99, P=0.032$, Table 3 and Figure 2). The studies on Caucasians and other populations demonstrated that there was no significant association between rs799917 polymorphism and ovarian cancer, Glioblastoma, SGC and CML in any genetic models (Table 3 and Figure 2). BRCA1 has been considered a risk-related gene for breast cancer, and the studies included in our metaanalysis reveal that the $B R C A 1$ rs799917 polymorphism plays an important role in the risk of non-breast cancer, especially in Asian populations. Due to the limited number of studies on each type of cancer, the conclusions drawn from these studies should be interpreted with caution. We require more studies on the association between rs799917 polymorphism and risk of these types of cancer to verify these conclusions, and the biological function of the polymorphism should be investigated as well.

No significant association was observed between rs799917 polymorphism and cancer risk in subgroup analyses based on control source and quality score (Table 3). 
Table 1: Characteristics of the studies included in the meta-analysis

\begin{tabular}{|c|c|c|c|c|c|c|}
\hline First author & Year & Country & Ethnicity & Cancer type & Genotyping method & $\begin{array}{l}\text { Control } \\
\text { source }\end{array}$ \\
\hline Dunning [14] & 1997 & UK & Caucasian & $\begin{array}{l}\text { Breast cancer } \\
\text { Ovarian cancer }\end{array}$ & ASOs hybridisation & PB \\
\hline Baynes [15] & 2007 & UK & $\begin{array}{l}\text { Caucasian ( } \\
>98 \%)\end{array}$ & Breast cancer & Taqman & PB \\
\hline Soucek [16] & 2007 & Czech Republic & Caucasian & Breast cancer & PCR-RFLP & HB \\
\hline Chang [17] & 2008 & USA & Caucasian & glioblastoma & ParAllele SNP panel & PB \\
\hline Wang [18] & 2009 & China & Asian & Breast cancer & PCR-PIRA & PB \\
\hline Huo [19] & 2009 & China & Asian & Breast cancer & PCR-PIRA & PB \\
\hline Zhou [20] & 2009 & China & Asian & Cervical cancer & PCR-PIRA & PB \\
\hline $\begin{array}{l}\text { Dombernowsky } \\
\text { [21] }\end{array}$ & 2009 & Denmark & Caucasian & Breast cancer & Taqman & PB \\
\hline Abbas [22] & 2010 & Germany & Caucasian & Breast cancer & MALDI-TOF MS & PB \\
\hline Nicoloso [23] & 2010 & USA & Caucasian & Breast cancer & $\begin{array}{c}\text { BigDye Terminator } \\
\text { Reaction }\end{array}$ & NR \\
\hline $\mathrm{Xu}[24]$ & 2012 & USA & Mix & SGC & PCR-RFLP & $\mathrm{HB}$ \\
\hline Zhang [25] & 2013 & China & Asian & ESCC & PCR-RFLP & $\mathrm{PB}$ \\
\hline Ricks-Santi [26] & 2013 & USA & Caucasian & Breast cancer & TaqMan & PB \\
\hline $\mathrm{Wu}[27]$ & 2013 & USA & Caucasian & Breast cancer & Taqman & FB \\
\hline Hasan [28] & 2013 & Saudi Arabia & Arab & Breast cancer & Taqman & HB \\
\hline Kim [29] & 2014 & Korea & Asian & NHL & PCR-PIRA & PB \\
\hline Wójcicka [30] & 2014 & Poland & Caucasian & PTC & iPLEX Gold system & PB \\
\hline Wang [31] & 2015 & China & Asian & Gastric cancer & PCR-RFLP & $\mathrm{PB}$ \\
\hline Gutierrez [32] & 2016 & Mexico & $\begin{array}{c}\text { Latin } \\
\text { American }\end{array}$ & CML & Taqman & Blood bank \\
\hline
\end{tabular}

SGC: Salivary Gland Carcinoma; ESCC: esophageal squamous cell carcinoma; NHL: Non-Hodgkin Lymphoma; PTC: Papillary Thyroid Carcinoma; CML: chronic myeloid leukemia; ASOs: allele-specific oligonucleotides; PCR-PIRA: PCR primer introduced restriction analysis assay; PCR-RFLP: PCR restriction fragment length polymorphism; MALDI-TOF MS: matrix-assisted laser desorption/ionization time-of-flight mass spectrometry; PB: population-based; HB: hospital-based; FB: family-based; NR: no record.

\section{Meta-analysis of rs1799950, rs1799966, and rs16941}

The main results of the meta-analysis for rs1799950, rs1799966, and rs16941 polymorphisms are shown in Table 4.

There were seven studies with 7,997 cases and 13,951 controls examining rs1799950 polymorphism. We found that rs 1799950 could decrease overall cancer risk (Table 4 and Figure 3). Furthermore, we found that rs 1799950 could decrease cancer risk among Caucasian populations in the subgroup analyses based on ethnicity and rs1799950 could decrease breast cancer risk in the subgroup analyses based on cancer type. Because all of the studies on breast cancer were conducted using Caucasian populations, we suggest that rs1799950 can decrease breast cancer risk among Caucasian populations in the homozygote and recessive models (GG vs. AA: OR, 0.46,
95\% Cl, 0.29-0.72, $P=0.001$; GG vs. AG + AA: OR, $0.46,95 \% \mathrm{Cl}, 0.29-0.72, P=0.001$, Table 4 and Figure 3 ).

There were six studies with 5,371 cases and 10,910 controls examining rs1799966 polymorphism. We did not find any association between rs1799966 and cancer risk, either in the overall analysis or in subgroup analyses (Table 4).

There were six studies with 3,672 cases and 7,591 controls examining rs 16941 polymorphism. We did not find association between rs16941 and overall cancer risk. However, in the subgroup analyses based on ethnicity, we found that rs16941 could increase cancer risk among Caucasian populations in the homozygote and recessive models (GG vs. AA: OR, 1.17, 95\% Cl, 1.07-1.35, $P=$ 0.033; GG vs. AG + AA: OR, 1.17, 95\% Cl, 1.01-1.33, $P$ $=0.033$, Table 4 and Figure 3 ). No significant association was observed between rs16941 polymorphism and cancer risk in the subgroup analyses based on cancer type. 
Table 2: BRCA1 polymorphisms genotype distribution and allele frequency in cases and controls

\begin{tabular}{|c|c|c|c|c|c|c|c|c|c|c|c|c|c|c|}
\hline \multirow[b]{3}{*}{ rs799917 } & \multicolumn{8}{|c|}{ Genotype $(N)$} & \multicolumn{4}{|c|}{ Allele frequency $(N)$} & \multirow{3}{*}{ HWE } & \multirow{3}{*}{ Score } \\
\hline & \multicolumn{4}{|c|}{ Case } & \multicolumn{4}{|c|}{ Control } & \multicolumn{2}{|c|}{ Case } & \multicolumn{2}{|c|}{ Control } & & \\
\hline & Total & $\mathrm{CC}$ & $\mathrm{CT}$ & TT & Total & $\mathrm{CC}$ & CT & TT & $\mathrm{C}$ & $\mathrm{T}$ & $\mathrm{C}$ & $\mathrm{T}$ & & \\
\hline Dunning [14](BC) & 801 & 342 & 370 & 89 & 572 & 266 & 250 & 56 & 1054 & 548 & 782 & 362 & 0.805 & 13 \\
\hline Dunning [14](OC) & 223 & 102 & 94 & 27 & 572 & 266 & 250 & 56 & 298 & 148 & 782 & 362 & 0.805 & 12 \\
\hline Chang [17] & 112 & 51 & 51 & 10 & 112 & 55 & 38 & 19 & 153 & 71 & 148 & 76 & 0.010 & 9 \\
\hline Wang [18] & 1004 & 381 & 483 & 140 & 1008 & 403 & 463 & 142 & 1245 & 763 & 1269 & 747 & 0.626 & 14 \\
\hline Huo [19] & 568 & 215 & 283 & 70 & 624 & 255 & 285 & 84 & 713 & 423 & 795 & 453 & 0.757 & 14 \\
\hline Zhou [20] & 404 & 166 & 196 & 42 & 404 & 158 & 183 & 63 & 528 & 280 & 499 & 309 & 0.410 & 13 \\
\hline Dombernowsky [21] & 1201 & 550 & 496 & 155 & 4120 & 1756 & 1896 & 467 & 1596 & 806 & 5408 & 2830 & 0.187 & 13 \\
\hline Abbas [22] & 3136 & 1403 & 1377 & 356 & 5470 & 2433 & 2396 & 641 & 4183 & 2089 & 7262 & 3678 & 0.168 & 13 \\
\hline Nicoloso [23] & 247 & 90 & 118 & 39 & 185 & 90 & 75 & 20 & 298 & 196 & 255 & 115 & 0.465 & 7 \\
\hline $\mathrm{Xu}[24]$ & 156 & 71 & 62 & 23 & 511 & 198 & 226 & 87 & 204 & 108 & 622 & 400 & 0.105 & 11 \\
\hline Zhang [25] & 1128 & 482 & 530 & 116 & 1150 & 444 & 524 & 182 & 1494 & 762 & 1412 & 888 & 0.188 & 13 \\
\hline $\mathrm{Wu}[27]$ & 335 & 108 & 164 & 63 & 408 & 120 & 211 & 77 & 380 & 290 & 451 & 365 & 0.354 & 12 \\
\hline Hasan [28] & 100 & 31 & 37 & 32 & 100 & 30 & 36 & 34 & 99 & 101 & 96 & 104 & 0.005 & 7 \\
\hline Kim [29] & 687 & 364 & 273 & 50 & 1700 & 828 & 715 & 157 & 1001 & 373 & 2371 & 1029 & 0.882 & 14 \\
\hline Wang [31] & 660 & 286 & 313 & 61 & 800 & 302 & 365 & 133 & 885 & 435 & 969 & 631 & 0.204 & 13 \\
\hline Gutierrez [32] & 312 & 147 & 129 & 36 & 469 & 200 & 210 & 59 & 423 & 201 & 610 & 328 & 0.737 & 12 \\
\hline rs1799950 & Total & $\mathrm{AA}$ & $\mathrm{AG}$ & GG & Total & $\mathrm{AA}$ & $\mathrm{AG}$ & GG & A & G & $\mathrm{A}$ & G & & \\
\hline Dunning [14] (BC) & 765 & 684 & 81 & 0 & 631 & 550 & 74 & 7 & 1449 & 81 & 1174 & 88 & 0.016 & 10 \\
\hline Dunning [14] (OC) & 230 & 195 & 35 & 0 & 631 & 550 & 74 & 7 & 425 & 35 & 1174 & 88 & 0.016 & 9 \\
\hline Baynes [15] & 2182 & 1955 & 221 & 6 & 2273 & 2004 & 256 & 13 & 4131 & 233 & 4264 & 282 & 0.125 & 15 \\
\hline Soucek [16] & 305 & 261 & 43 & 1 & 305 & 243 & 56 & 6 & 565 & 45 & 542 & 68 & 0.201 & 11 \\
\hline Dombernowsky [21] & 1200 & 1048 & 147 & 5 & 4119 & 3589 & 513 & 17 & 2243 & 157 & 7691 & 547 & 0.771 & 13 \\
\hline Abbas[22] & 3139 & 2711 & 417 & 11 & 5481 & 4762 & 679 & 40 & 5839 & 439 & 10203 & 759 & 0.004 & 10 \\
\hline $\mathrm{Xu}[24]$ & 156 & 132 & 24 & 0 & 511 & 455 & 56 & 0 & 288 & 24 & 966 & 56 & 0.190 & 11 \\
\hline rs1799966 & Total & AA & $\mathrm{AG}$ & GG & Total & $\mathrm{AA}$ & $\mathrm{AG}$ & GG & $\mathrm{A}$ & G & A & G & & \\
\hline Soucek[16] & 449 & 270 & 146 & 33 & 295 & 127 & 132 & 36 & 686 & 212 & 386 & 204 & 0.851 & 11 \\
\hline Chang[17] & 111 & 53 & 49 & 9 & 112 & 56 & 39 & 17 & 155 & 67 & 151 & 73 & 0.028 & 9 \\
\hline Dombernowsky [21] & 1198 & 557 & 508 & 133 & 4119 & 1850 & 1834 & 435 & 1622 & 774 & 5534 & 2704 & 0.535 & 13 \\
\hline Abbas [22] & 3140 & 1422 & 1365 & 353 & 5487 & 2445 & 2391 & 651 & 4209 & 2071 & 7281 & 3693 & 0.073 & 13 \\
\hline $\mathrm{Xu}$ [24] & 156 & 80 & 62 & 14 & 511 & 229 & 233 & 49 & 222 & 90 & 691 & 331 & 0.353 & 11 \\
\hline $\mathrm{Wu}$ [27] & 317 & 132 & 143 & 42 & 386 & 162 & 182 & 42 & 407 & 227 & 506 & 266 & 0.388 & 12 \\
\hline rs16941 & Total & $\mathrm{AA}$ & $\mathrm{AG}$ & GG & Total & $\mathrm{AA}$ & $\mathrm{AG}$ & GG & A & G & A & G & & \\
\hline Soucek [16] & 305 & 130 & 142 & 33 & 305 & 138 & 131 & 36 & 402 & 208 & 407 & 203 & 0.567 & 11 \\
\hline Chang [17] & 110 & 51 & 48 & 11 & 109 & 55 & 36 & 18 & 150 & 70 & 146 & 72 & 0.008 & 9 \\
\hline Dombernowsky [21] & 1199 & 563 & 491 & 145 & 4120 & 1854 & 1835 & 431 & 1617 & 781 & 5543 & 2697 & 0.463 & 13 \\
\hline $\mathrm{Xu}[24]$ & 156 & 81 & 61 & 14 & 511 & 230 & 227 & 54 & 223 & 89 & 687 & 335 & 0.856 & 11 \\
\hline Ricks-Santi [26] & 267 & 121 & 124 & 22 & 525 & 255 & 227 & 43 & 366 & 168 & 737 & 313 & 0.446 & 12 \\
\hline Wójcicka [30] & 1635 & 807 & 667 & 161 & 2021 & 1074 & 792 & 155 & 2281 & 989 & 2940 & 1102 & 0.592 & 14 \\
\hline
\end{tabular}

HWE: Hardy-Weinberg equilibrium.

\section{Sensitivity analysis}

We assessed sensitivity by omitting a single study each time. The sensitivity analysis of rs799917 showed that our data were stable in the heterozygote and dominant genetic models but unstable in the allele, homozygote and recessive models (Figure 4 and
Supplementary Table 2). In the allele and homozygote models, after omitting the study by Dunning et al. (BC) or Nicoloso et al., rs799917 was found to significantly decrease cancer risk, and in the recessive model, after omitting the study by Dunning et al. (OC), Dombernowsky et al., or Nicoloso et al., rs799917 was found to significantly decrease cancer risk. 
Table 3: Meta-analysis of the association between rs799917 polymorphism and cancer risk

\begin{tabular}{|c|c|c|c|c|c|c|c|c|c|c|c|c|c|c|c|c|}
\hline \multirow[t]{2}{*}{ Subgroup } & \multirow[t]{2}{*}{ No. } & \multicolumn{3}{|c|}{ T vs. C } & \multicolumn{3}{|c|}{ TT vs. CC } & \multicolumn{3}{|c|}{ CT vs. CC } & \multicolumn{3}{|c|}{ TT + CT vs. CC } & \multicolumn{3}{|c|}{ TT vs. $\mathrm{CT}+\mathrm{CC}$} \\
\hline & & $\begin{array}{c}\text { OR } \\
(95 \% \mathrm{Cl})\end{array}$ & POR & $\mathbf{P h}$ & OR $(95 \% \mathrm{Cl})$ & POR & $\mathbf{P h}$ & $\begin{array}{c}\text { OR } \\
(95 \% \mathrm{Cl})\end{array}$ & POR & $\mathbf{P h}$ & $\begin{array}{c}\text { OR } \\
(95 \% \mathrm{Cl})\end{array}$ & POR & Ph & $\begin{array}{c}\text { OR } \\
(95 \% \mathrm{Cl})\end{array}$ & POR & Ph \\
\hline Overall & 16 & $\begin{array}{c}0.95(0.89- \\
1.01)^{*}\end{array}$ & 0.106 & 0.001 & $\begin{array}{c}0.87 \\
(0.75-1.02)^{*}\end{array}$ & 0.080 & $\begin{array}{c}< \\
0.001\end{array}$ & $\begin{array}{c}0.98(0.91- \\
1.05)^{*}\end{array}$ & 0.533 & 0.078 & $\begin{array}{c}0.96 \\
(0.89-1.03)^{*}\end{array}$ & 0.241 & 0.022 & $\begin{array}{c}0.88(0.77- \\
1.01)^{*}\end{array}$ & 0.078 & $<0.001$ \\
\hline \multicolumn{17}{|l|}{ Ethnicity } \\
\hline Caucasian & 7 & $\begin{array}{c}1.00 \\
(0.96-1.05)\end{array}$ & 0.901 & 0.111 & $\begin{array}{c}1.03 \\
(0.93-1.14)\end{array}$ & 0.608 & 0.187 & $\begin{array}{c}1.02(0.89- \\
1.17)^{*}\end{array}$ & 0.801 & 0.023 & $\begin{array}{c}1.03 \\
(0.91-1.16)^{*}\end{array}$ & 0.680 & 0.041 & $\begin{array}{c}1.04 \\
(0.94-1.15)\end{array}$ & 0.419 & 0.196 \\
\hline Asian & 6 & $\begin{array}{c}0.89(0.80- \\
0.99)^{*}\end{array}$ & 0.032 & 0.006 & $\begin{array}{c}0.72 \\
(0.56-0.93)^{*}\end{array}$ & 0.011 & 0.003 & $\begin{array}{c}0.98 \\
(0.90-1.07)\end{array}$ & 0.653 & 0.291 & $\begin{array}{c}0.92 \\
(0.82-1.04)^{*}\end{array}$ & 0.194 & 0.064 & $\begin{array}{c}0.72(0.58- \\
0.89)^{*}\end{array}$ & 0.003 & 0.013 \\
\hline Others & 3 & $\begin{array}{c}0.87 \\
(0.75-1.02)\end{array}$ & 0.078 & 0.842 & $\begin{array}{c}0.81 \\
(0.59-1.11)\end{array}$ & 0.189 & 0.887 & $\begin{array}{c}0.83 \\
(0.66-1.04)\end{array}$ & 0.101 & 0.802 & $\begin{array}{c}0.82 \\
(0.67-1.01)\end{array}$ & 0.067 & 0.802 & $\begin{array}{c}0.89 \\
(0.66-1.18)\end{array}$ & 0.410 & 0.971 \\
\hline \multicolumn{17}{|l|}{ Cancer type } \\
\hline Breast cancer & 8 & $\begin{array}{c}1.01 \\
(0.97-1.05)\end{array}$ & 0.716 & 0.171 & $\begin{array}{c}1.03 \\
(0.93-1.13)\end{array}$ & 0.607 & 0.484 & $\begin{array}{c}1.03(0.92- \\
1.16)^{*}\end{array}$ & 0.571 & 0.023 & $\begin{array}{c}1.04 \\
(0.93-1.15)^{*}\end{array}$ & 0.484 & 0.037 & $\begin{array}{c}1.03 \\
(0.94-1.12)\end{array}$ & 0.592 & 0.607 \\
\hline (Caucasian) & 5 & $\begin{array}{c}1.03(0.94- \\
1.13)^{*}\end{array}$ & 0.522 & 0.045 & $\begin{array}{c}1.03 \\
(0.92-1.15)\end{array}$ & 0.604 & 0.176 & $\begin{array}{c}1.01(0.86- \\
1.18)^{*}\end{array}$ & 0.951 & 0.012 & $\begin{array}{c}1.03 \\
(0.89-1.19)^{*}\end{array}$ & 0.732 & 0.013 & $\begin{array}{c}1.05 \\
(0.95-1.16)\end{array}$ & 0.383 & 0.340 \\
\hline (Asian) & 2 & $\begin{array}{c}1.04 \\
(0.94-1.15)\end{array}$ & 0.436 & 1.000 & $\begin{array}{c}1.02 \\
(0.82-1.27)\end{array}$ & 0.838 & 0.818 & $\begin{array}{c}1.13 \\
(0.97-1.31)\end{array}$ & 0.108 & 0.680 & $\begin{array}{c}1.11 \\
(0.96-1.28)\end{array}$ & 0.165 & 0.785 & $\begin{array}{c}0.96 \\
(0.78-1.17)\end{array}$ & 0.672 & 0.678 \\
\hline (Arab) & 1 & $\begin{array}{c}0.94 \\
(0.64-1.39)\end{array}$ & 0.764 & --- & $\begin{array}{c}0.92 \\
(0.45-1.83)\end{array}$ & 0.793 & --- & $\begin{array}{c}1.00 \\
(0.50-1.96)\end{array}$ & 0.988 & --- & $\begin{array}{c}0.95 \\
(0.52-1.74)\end{array}$ & 0.878 & --- & $\begin{array}{c}0.91 \\
(0.51-1.65)\end{array}$ & 0.764 & ---- \\
\hline \multicolumn{17}{|l|}{$\begin{array}{c}\text { Other } \\
\text { cancers }\end{array}$} \\
\hline \multicolumn{17}{|l|}{ (Asian) } \\
\hline $\begin{array}{c}\text { Cervical } \\
\text { cancer }\end{array}$ & 1 & $\begin{array}{c}0.86 \\
(0.70-1.05)\end{array}$ & 0.134 & --- & $\begin{array}{c}0.64 \\
(0.41-0.99)\end{array}$ & 0.046 & --- & $\begin{array}{c}1.02 \\
(0.76-1.37)\end{array}$ & 0.899 & --- & $\begin{array}{c}0.92 \\
(0.70-1.22)\end{array}$ & 0.566 & --- & $\begin{array}{c}0.63 \\
(0.41-0.95)\end{array}$ & 0.029 & ---- \\
\hline ESCC & 1 & $\begin{array}{c}0.81 \\
(0.72-0.92)\end{array}$ & 0.001 & --- & $\begin{array}{c}0.59 \\
(0.45-0.77)\end{array}$ & $<0.001$ & --- & $\begin{array}{c}0.93 \\
(0.78-1.11)\end{array}$ & 0.433 & --- & $\begin{array}{c}0.84 \\
(0.71-1.00)\end{array}$ & 0.045 & --- & $\begin{array}{c}0.61 \\
(0.48-0.78)\end{array}$ & $\begin{array}{c}< \\
0.001\end{array}$ & --- \\
\hline $\begin{array}{l}\text { Gastric } \\
\text { cancer }\end{array}$ & 1 & $\begin{array}{c}0.76 \\
(0.65-0.88)\end{array}$ & $<0.001$ & --- & $\begin{array}{c}0.48 \\
(0.34-0.68)\end{array}$ & $<0.001$ & --- & $\begin{array}{c}0.91 \\
(0.73-1.13)\end{array}$ & 0.379 & --- & $\begin{array}{c}0.79 \\
(0.64-0.98)\end{array}$ & 0.030 & --- & $\begin{array}{c}0.51 \\
(0.37-0.71)\end{array}$ & $\begin{array}{c}< \\
0.001\end{array}$ & --- \\
\hline NHL & 1 & $\begin{array}{c}0.86 \\
(0.75-0.99)\end{array}$ & 0.032 & --- & $\begin{array}{c}0.72 \\
(0.52-1.02)\end{array}$ & 0.064 & --- & $\begin{array}{c}0.87 \\
(0.72-1.05)\end{array}$ & 0.138 & --- & $\begin{array}{c}0.84 \\
(0.71-1.01)\end{array}$ & 0.059 & --- & $\begin{array}{c}0.77 \\
(0.55-1.08)\end{array}$ & 0.125 & --- \\
\hline \multicolumn{17}{|l|}{ (Caucasian) } \\
\hline $\begin{array}{c}\text { Ovarian } \\
\text { cancer }\end{array}$ & 1 & $\begin{array}{c}1.07 \\
(0.85-1.36)\end{array}$ & 0.554 & --- & $\begin{array}{c}1.26 \\
(0.75-2.10)\end{array}$ & 0.381 & --- & $\begin{array}{c}0.98 \\
(0.71-1.36)\end{array}$ & 0.907 & --- & $\begin{array}{c}1.03 \\
(0.76-1.41)\end{array}$ & 0.846 & --- & $\begin{array}{c}1.27 \\
(0.78-2.07)\end{array}$ & 0.338 & ---- \\
\hline Glioblastoma & 1 & $\begin{array}{c}0.90 \\
(0.61-1.34)\end{array}$ & 0.615 & ---- & $\begin{array}{c}0.57 \\
(0.24-1.34)\end{array}$ & 0.194 & ---- & $\begin{array}{c}1.45 \\
(0.82-2.55)\end{array}$ & 0.201 & ---- & $\begin{array}{c}1.15 \\
(0.68-1.95)\end{array}$ & 0.593 & --- & $\begin{array}{c}0.48 \\
(0.21-1.09)\end{array}$ & 0.078 & --- \\
\hline \multicolumn{17}{|l|}{$\begin{array}{c}\text { (other } \\
\text { ethnicities) }\end{array}$} \\
\hline $\mathrm{SGC}$ & 1 & $\begin{array}{c}0.82 \\
(0.63-1.07)\end{array}$ & 0.150 & --- & $\begin{array}{c}0.74 \\
(0.43-1.26)\end{array}$ & 0.263 & --- & $\begin{array}{c}0.77 \\
(0.52-1.13)\end{array}$ & 0.179 & --- & $\begin{array}{c}0.76 \\
(0.53-1.09)\end{array}$ & 0.132 & --- & $\begin{array}{c}0.84 \\
(0.51-1.39)\end{array}$ & 0.502 & --- \\
\hline CML & 1 & $\begin{array}{c}0.88 \\
(0.71-1.10)\end{array}$ & 0.260 & --- & $\begin{array}{c}0.83 \\
(0.52-1.32)\end{array}$ & 0.434 & ---- & $\begin{array}{c}0.84 \\
(0.62-1.14)\end{array}$ & 0.250 & --- & $\begin{array}{c}0.84 \\
(0.63-1.11)\end{array}$ & 0.218 & --- & $\begin{array}{c}0.91 \\
(0.58-1.41)\end{array}$ & 0.663 & ---- \\
\hline \multicolumn{17}{|c|}{ Control source } \\
\hline PB & 11 & $\begin{array}{c}0.94(0.88- \\
1.01)^{*}\end{array}$ & 0.089 & 0.002 & $\begin{array}{c}0.85 \\
(0.71-1.01)^{*}\end{array}$ & 0.065 & $<0.001$ & $\begin{array}{c}0.97 \\
(0.92-1.03)\end{array}$ & 0.343 & 0.126 & $\begin{array}{c}0.96 \\
(0.89-1.03)^{*}\end{array}$ & 0.236 & 0.064 & $\begin{array}{c}0.84(0.71- \\
1.00)^{*}\end{array}$ & 0.056 & $\begin{array}{c}< \\
0.001\end{array}$ \\
\hline HB & 2 & $\begin{array}{c}0.86 \\
(0.69-1.07)\end{array}$ & 0.172 & 0.578 & $\begin{array}{c}0.80 \\
(0.52-1.21)\end{array}$ & 0.289 & 0.637 & $\begin{array}{c}0.82 \\
(0.58-1.15)\end{array}$ & 0.240 & 0.512 & $\begin{array}{c}0.81 \\
(0.59-1.10)\end{array}$ & 0.172 & 0.520 & $\begin{array}{c}0.87 \\
(0.60-1.27)\end{array}$ & 0.477 & 0.838 \\
\hline others & 3 & $\begin{array}{c}1.05(0.80- \\
1.38)^{*}\end{array}$ & 0.725 & 0.016 & $\begin{array}{c}1.09(0.68- \\
1.74)^{*}\end{array}$ & 0.720 & 0.070 & $\begin{array}{c}1.02(0.71- \\
1.47)^{*}\end{array}$ & 0.913 & 0.035 & $\begin{array}{c}1.05 \\
(0.71-1.55)^{*}\end{array}$ & 0.824 & 0.013 & $\begin{array}{c}1.05 \\
(0.82-1.36)\end{array}$ & 0.681 & 0.326 \\
\hline \multicolumn{17}{|c|}{ Quality score } \\
\hline$\geq 12$ & 12 & $\begin{array}{c}0.94(0.88- \\
1.00)^{*}\end{array}$ & 0.054 & 0.003 & $\begin{array}{c}0.86(0.73- \\
1.01)^{*}\end{array}$ & 0.063 & $<0.001$ & $\begin{array}{c}0.96 \\
(0.91-1.02)\end{array}$ & 0.166 & 0.202 & $\begin{array}{c}0.94 \\
(0.88-1.01)^{*}\end{array}$ & 0.099 & 0.081 & $\begin{array}{c}0.87(0.75- \\
1.02)^{*}\end{array}$ & 0.085 & $<0.001$ \\
\hline$<12$ & 4 & $\begin{array}{c}1.01(0.76- \\
1.35)^{*}\end{array}$ & 0.930 & 0.028 & $\begin{array}{c}0.96(0.57- \\
1.61)^{*}\end{array}$ & 0.864 & 0.057 & $\begin{array}{c}1.14(0.78- \\
1.68)^{*}\end{array}$ & 0.496 & 0.066 & $\begin{array}{c}1.09 \\
(0.74-1.60)^{*}\end{array}$ & 0.672 & 0.036 & $\begin{array}{c}0.94 \\
(0.70-1.25)\end{array}$ & 0.662 & 0.129 \\
\hline
\end{tabular}

SGC: Salivary Gland Carcinoma; ESCC: esophageal squamous cell carcinoma; NHL: Non-Hodgkin Lymphoma; PTC: Papillary Thyroid Carcinoma; CML: chronic myeloid leukemia; OR, odds ratio; 95\% $\mathrm{CI}, 95 \%$ confidence interval; $P \mathrm{OR}$, pool $P$ value; $P \mathrm{~h}, P$ value of heterogeneity test; "indicates that the $\mathrm{OR}, 95 \% \mathrm{Cl}$, and corresponding $P \mathrm{OR}$ were calculated based on the random-effects model; otherwise, the fixed-effects model was used.

No individual study showed a significant influence on the pooled ORs for rs1799950 and rs1799966 (Supplementary Table 3), indicating that our data were relatively stable for these polymorphisms

The sensitivity analysis of rs 16941 showed that the results were stable in the allele, heterozygote, and dominant models but unstable in the homozygote and recessive models (Supplementary Table 3). In the homozygote model, after omitting the study by Chang et al. or Xu et al. rs16941 was shown to increase cancer risk, and in the homozygote model, after omitting the study by Soucek et al., Chang et al., Xu et al. or Ricks-Santi et al. rs16941 was shown to increase cancer risk. 
Table 4: Meta-analysis of the association between rs1799950, rs1799966, and rs16941 polymorphisms and cancer risk

\begin{tabular}{|c|c|c|c|c|c|c|c|c|c|c|c|c|c|c|c|c|}
\hline \multirow[t]{2}{*}{ Subgroup } & \multirow[t]{2}{*}{ No. } & \multicolumn{3}{|c|}{ G vs. A } & \multicolumn{3}{|c|}{ GG vs. AA } & \multicolumn{3}{|c|}{ AG vs. AA } & \multicolumn{3}{|c|}{ GG+AG vs. AA } & \multicolumn{3}{|c|}{ GG vs. AG+AA } \\
\hline & & $\begin{array}{c}\text { OR } \\
(95 \% \mathrm{Cl})\end{array}$ & $\mathbf{P Z}$ & $\mathbf{P h}$ & OR $(95 \% \mathrm{Cl})$ & $\mathbf{P Z}$ & Ph & OR $(95 \% \mathrm{Cl})$ & $\mathbf{P Z}$ & Ph & $\begin{array}{c}\text { OR } \\
(95 \% \mathrm{Cl})\end{array}$ & $\mathbf{P Z}$ & $\mathbf{P h}$ & OR $(95 \% \mathrm{Cl})$ & $\mathbf{P Z}$ & Ph \\
\hline \multicolumn{17}{|l|}{ rs1799950 } \\
\hline Overall & 7 & $\begin{array}{c}0.93(0.81- \\
1.06)^{*}\end{array}$ & 0.257 & 0.062 & $\begin{array}{c}0.44 \\
(0.28-0.69) \\
\text { (excluded } \\
\text { Xu) }\end{array}$ & $<0.001$ & 0.300 & $\begin{array}{c}1.00 \\
(0.92-1.09)\end{array}$ & 0.941 & 0.140 & $\begin{array}{c}0.96 \\
(0.84 \\
1.09)^{*}\end{array}$ & 0.535 & 0.095 & $\begin{array}{l}0.44(0.28-0.69) \\
(\text { excluded Xu) }\end{array}$ & $<0.001$ & 0.308 \\
\hline \multicolumn{17}{|l|}{ Ethnicity } \\
\hline Caucasian & 6 & $\begin{array}{c}0.94(0.86- \\
1.01)\end{array}$ & 0.105 & 0.101 & $\begin{array}{c}0.44 \\
(0.28-0.69)\end{array}$ & $<0.001$ & 0.300 & $\begin{array}{c}0.99 \\
(0.91-1.08)\end{array}$ & 0.873 & 0.190 & $\begin{array}{c}0.96 \\
(0.88- \\
1.05)\end{array}$ & 0.377 & 0.144 & $0.44(0.28-0.69)$ & $<0.001$ & 0.308 \\
\hline Mix & 1 & $\begin{array}{c}1.44(0.88- \\
2.36)\end{array}$ & 0.152 & --- & --- & --- & ---- & $\begin{array}{c}1.48 \\
(0.88-2.48)\end{array}$ & 0.138 & --- & $\begin{array}{c}1.48 \\
(0.88- \\
2.48)\end{array}$ & 0.138 & --- & --- & --- & --- \\
\hline \multicolumn{17}{|l|}{$\begin{array}{l}\text { Cancer } \\
\text { type }\end{array}$} \\
\hline $\begin{array}{l}\text { Breast } \\
\text { cancer }\end{array}$ & 5 & $\begin{array}{c}0.89(0.78- \\
1.02)^{*}\end{array}$ & 0.088 & 0.072 & $\begin{array}{c}0.46 \\
(0.29-0.72)\end{array}$ & 0.001 & 0.233 & $\begin{array}{c}0.98 \\
(0.90-1.07)\end{array}$ & 0.675 & 0.231 & $\begin{array}{c}0.95 \\
(0.87- \\
1.04)\end{array}$ & 0.280 & 0.135 & $0.46(0.29-0.72)$ & 0.001 & 0.244 \\
\hline $\begin{array}{l}\text { other } \\
\text { cancers }\end{array}$ & 2 & $\begin{array}{c}1.22(0.89- \\
1.67)\end{array}$ & 0.216 & 0.411 & $\begin{array}{c}0.19(0.01-3 . \\
30)\end{array}$ & 0.253 & ---- & $\begin{array}{c}1.39 \\
(1.00-1.94)\end{array}$ & 0.052 & 0.767 & $\begin{array}{c}1.32 \\
(0.95- \\
1.83)\end{array}$ & 0.103 & 0.574 & $0.18(0.01-3.18)$ & 0.242 & --- \\
\hline & & & & & $\begin{array}{l}\text { (excluded } \\
\text { Xu) }\end{array}$ & & & & & & & & & (excluded Xu) & & \\
\hline \multicolumn{17}{|l|}{ rs1799966 } \\
\hline Overall & 6 & $\begin{array}{c}0.89(0.78- \\
1.02)^{*}\end{array}$ & 0.091 & 0.001 & $\begin{array}{c}0.86 \\
(0.69-1.09)^{*}\end{array}$ & 0.207 & 0.038 & $\begin{array}{c}0.87 \\
(0.73-1.03)^{*}\end{array}$ & 0.112 & 0.005 & $\begin{array}{c}0.86 \\
(0.72- \\
1.02)^{*}\end{array}$ & 0.087 & 0.002 & $0.95(0.86-1.06)$ & 0.372 & 0.119 \\
\hline \multicolumn{17}{|l|}{ Ethnicity } \\
\hline Caucasian & 5 & $\begin{array}{c}0.90(0.78- \\
1.04)^{*}\end{array}$ & 0.148 & 0.001 & $\begin{array}{c}0.86 \\
(0.67-1.11)^{*}\end{array}$ & 0.252 & 0.020 & $\begin{array}{c}0.89 \\
(0.73-1.07)^{*}\end{array}$ & 0.206 & 0.003 & $\begin{array}{l}0.87 \\
(0.72- \\
1.06)^{*}\end{array}$ & 0.161 & 0.001 & $0.92\left(\begin{array}{c}0.75-1.14) \\
*\end{array}\right.$ & 0.445 & 0.068 \\
\hline Mix & 1 & $\begin{array}{c}0.85(0.64- \\
1.12)\end{array}$ & 0.239 & --- & $\begin{array}{c}0.82 \\
(0.43-1.56)\end{array}$ & 0.542 & ---- & $\begin{array}{c}0.76 \\
(0.52-1.11)\end{array}$ & 0.159 & --- & $\begin{array}{r}0.77 \\
(0.54 \\
1.11)\end{array}$ & 0.157 & --- & $0.93(0.50-1.73)$ & 0.818 & --- \\
\hline \multicolumn{17}{|l|}{$\begin{array}{l}\text { Cancer } \\
\text { type }\end{array}$} \\
\hline $\begin{array}{l}\text { Breast } \\
\text { cancer }\end{array}$ & 4 & $\begin{array}{c}0.90(0.76- \\
1.05)^{*}\end{array}$ & 0.180 & $<0.001$ & $\begin{array}{c}0.89 \\
(0.68-1.16)^{*}\end{array}$ & 0.382 & 0.016 & $\begin{array}{c}0.85 \\
(0.70-1.04)^{*}\end{array}$ & 0.114 & 0.002 & $\begin{array}{c}0.85 \\
(0.69- \\
1.05)^{*}\end{array}$ & 0.127 & $<0.001$ & $\begin{array}{c}0.96(0.79-1.17) \\
*\end{array}$ & 0.665 & 0.093 \\
\hline $\begin{array}{l}\text { Other } \\
\text { cancers }\end{array}$ & 2 & $\begin{array}{c}0.86(0.69- \\
1.08)\end{array}$ & 0.200 & 0.825 & $\begin{array}{c}0.72 \\
(0.43-1.21)\end{array}$ & 0.215 & 0.499 & $\begin{array}{c}0.91 \\
(0.66-1.24)\end{array}$ & 0.533 & 0.109 & $\begin{array}{r}0.86 \\
(0.64 \\
1.16)\end{array}$ & 0.281 & 0.328 & $0.74(0.45-1.23)$ & 0.251 & 0.240 \\
\hline \multicolumn{17}{|l|}{ rs 16941} \\
\hline Overall & 6 & $\begin{array}{c}1.05(0.99- \\
1.12)\end{array}$ & 0.130 & 0.147 & $\begin{array}{c}1.14 \\
(0.99-1.31)\end{array}$ & 0.064 & 0.271 & $\begin{array}{c}1.03 \\
(0.88-1.19)^{\star}\end{array}$ & 0.734 & 0.055 & $\begin{array}{l}1.03 \\
(0.90- \\
1.18)^{*}\end{array}$ & 0.642 & 0.082 & $1.14(1.00-1.31)$ & 0.052 & 0.257 \\
\hline \multicolumn{17}{|l|}{ Ethnicity } \\
\hline Caucasian & 5 & $\begin{array}{c}1.06(1.00- \\
1.13)\end{array}$ & 0.059 & 0.293 & $\begin{array}{c}1.17 \\
(1.01-1.35)\end{array}$ & 0.033 & 0.346 & $\begin{array}{c}1.06 \\
(0.91-1.24)^{*}\end{array}$ & 0.444 & 0.072 & $\begin{array}{c}1.05 \\
(0.97- \\
1.14)\end{array}$ & 0.233 & 0.150 & $1.17(1.01-1.33)$ & 0.033 & 0.241 \\
\hline Mix & 1 & $\begin{array}{c}0.82(0.62- \\
1.08)\end{array}$ & 0.158 & ---- & $\begin{array}{c}0.74 \\
(0.39-1.40)\end{array}$ & & --- & $\begin{array}{c}0.76 \\
(0.52-1.12)\end{array}$ & 0.162 & --- & $\begin{array}{c}0.76 \\
(0.53- \\
1.09)\end{array}$ & 0.130 & --- & $0.83(0.45-1.55)$ & 0.565 & ---- \\
\hline \multicolumn{17}{|l|}{$\begin{array}{l}\text { Cancer } \\
\text { type }\end{array}$} \\
\hline $\begin{array}{l}\text { Breast } \\
\text { cancer }\end{array}$ & 3 & $\begin{array}{c}1.01(0.93- \\
1.10)\end{array}$ & 0.820 & 0.772 & $\begin{array}{c}1.09 \\
(0.90-1.31)\end{array}$ & 0.376 & 0.905 & $\begin{array}{c}0.95 \\
(0.84-1.06)\end{array}$ & 0.356 & 0.143 & $\begin{array}{c}0.97 \\
(0.87- \\
1.09)\end{array}$ & 0.634 & 0.304 & $1.12(0.94-1.34)$ & 0.208 & 0.584 \\
\hline $\begin{array}{l}\text { Other } \\
\text { cancers }\end{array}$ & 3 & $\begin{array}{c}1.00(0.78- \\
1.27)^{*}\end{array}$ & 0.970 & 0.055 & $\begin{array}{c}0.97 \\
(0.57-1.64)^{*}\end{array}$ & 0.909 & 0.064 & $\begin{array}{c}1.09 \\
(0.96-1.23)\end{array}$ & 0.196 & 0.109 & $\begin{array}{l}1.03 \\
(0.77- \\
1.37)^{*}\end{array}$ & 0.864 & 0.087 & $\begin{array}{c}0.95(0.58-1.57) \\
*\end{array}$ & 0.840 & 0.069 \\
\hline
\end{tabular}

OR, odds ratio; $95 \% \mathrm{CI}, 95 \%$ confidence interval; $P \mathrm{OR}$, pool $P$ value; $P \mathrm{~h}, P$ value of heterogeneity test; $*$ indicates that the $\mathrm{OR}, 95 \% \mathrm{Cl}$, and corresponding $P \mathrm{OR}$ were calculated based on the random-effects model; otherwise, the fixed-effects model was used. 
Table 5: Publication bias analysis

\begin{tabular}{lccccc}
\hline Polymorphism & Genetic model & & Egger's test & & Begg's test \\
\hline rs799917 & & $\mathrm{t}$ & $95 \% \mathrm{Cl}$ & $P$ & $P$ \\
& T vs. C & -0.08 & $-2.042-1.886$ & 0.934 & 0.964 \\
TT vs. CC & -0.46 & $-2.574-1.659$ & 0.650 & 0.822 \\
CT vs. CC & 0.80 & $-0.915-1.998$ & 0.439 & 0.444 \\
TT + CT vs. CC & 0.39 & $-1.331-1.929$ & 0.700 & 0.444 \\
Ts1799950 & Ts. CT+CC & -0.70 & $-2.805-1.429$ & 0.497 & 1.000 \\
& G vs. A & -0.41 & $-3.838-2.777$ & 0.697 & 1.000 \\
GG vs. AA & -1.90 & $-3.500-0.657$ & 0.130 & 0.260 \\
AG vs. AA & -0.03 & $-3.051-2.983$ & 0.978 & 1.000 \\
rs1799966 & GG+AG vs. AA & -0.23 & $-3.455-2.888$ & 0.827 & 0.764 \\
& GG vs. AG+AA & -1.87 & $-3.478-0.677$ & 0.135 & 0.452 \\
G vs. A & -1.09 & $-5.522-2.416$ & 0.338 & 0.260 \\
GG vs. AA & -1.04 & $-4.217-1.911$ & 0.355 & 0.452 \\
AG vs. AA & -0.84 & $-5.071-2.705$ & 0.446 & 1.000 \\
GG+AG vs. AA & -0.98 & $-5.465-2.606$ & 0.381 & 1.000 \\
rs16941 & GG vs. AG+AA & -0.88 & $-3.586-1.853$ & 0.426 & 0.707 \\
G vs. A & -0.90 & $-4.186-2.131$ & 0.417 & 0.452 \\
GG vs. AA & -2.21 & $-3.645-0.413$ & 0.092 & 0.260 \\
& AG vs. AA & 0.50 & $-3.152-4.537$ & 0.643 & 1.000 \\
& GG+AG vs. AA & -0.04 & $-3.824-3.709$ & 0.968 & 1.000 \\
GG vs. AG+AA & -4.12 & $-3.323--0.647$ & 0.015 & 0.06 \\
\hline
\end{tabular}

\section{Publication bias}

Both Begg's test and Egger's test were performed to assess the publication bias of the studies of these SNPs. Our data revealed that there was no obvious publication bias in any of the five models for rs799917, rs 1799950 and rs1799966 (Table 5). There was publication bias in the recessive model for rs16941 (Egger's test $P=0.015$, Table 5).

The results of the sensitivity and publication bias analyses suggest that the number of studies included in our study was insufficient, especially for rs16941. Thus, the conclusions obtained by the current research should be interpreted with caution, and more studies are required to verify these conclusions in the future.

\section{DISCUSSION}

Based on GLOBOCAN estimates, about 14.1 million new cancer cases and 8.2 million deaths occurred worldwide in 2012 [2]. Cancer is the result of a combination of genetic and environmental pressures; familial cancer is primarily based on hereditary factors, and environmental factors are the main cause of sporadic cancer [1]. Thus, genetic and environmental factors are both important in cancer research, including the study of association between SNPs and cancer.

$B R C A 1$ is a tumor suppressor gene, and its most important function is DNA repair. Thus far, many studies on $B R C A 1$ have been concerned with the risk of breast and ovarian cancer in BRCA1 mutation carriers. There are several mutation types that lead to the protein inactivation of BRCA1, including frameshift, missense, nonsense, and splice mutations [13]. The mutation types tend to correspond with various populations and ethnicities [34]. Moreover, many studies have reported that BRCA1 SNP is associated with cancer risk. For example, using data from Genome-Wide Association Study (GWAS), Li et al. investigated the associations between DNA repair pathway genes and the risk of ESCC and GC, finding BRCA1 rs8176257 to be significantly associated with the risk of ESCC in a Han Chinese population [35]. In addition, Mullany et al. identified 327 SNPs that may play important roles in the regulation of miRNA expression based on the GWAS data. Two of these SNPs were significantly associated with colon cancer, one being BRCA1 8176318 [36]. To investigate the relationship between $B R C A 1$ SNPs and cancer risk, we conducted this meta-analysis.

Four BRCA1 gene polymorphisms were evaluated: rs799917, rs1799950, rs1799966, and rs16941. The SNPs for the meta-analysis were selected using the following criteria: the SNP was reported to be associated with 
cancer risk in previous studies, the SNPs were nonsynonymous, and the minor allele frequencies (MAFs) of the polymorphisms were greater than $1 \%$ in most of the populations from the 1000 Genomes Project Phase 3 (Supplementary Table 4). The linkage disequilibrium (LD) of the selected SNP is shown in Supplementary Figure 1.

rs799917 is a nonsynonymous SNP, and the rs799917 $\mathrm{C}>\mathrm{T}$ variant leads to an amino acid change from proline to leucine at position 871 in the BRCA1 protein. This amino acid change may impact the interaction between miR-638 and BRCA1 mRNA, leading to increased BRCA1 expression in rs799917 $\mathrm{T}$ allele carriers $[23,25]$. In the meta-analysis, we revealed that rs799917 polymorphism could decrease the risk of several types of non-breast cancer. Thus, this study and previous studies have shown that rs799917 is not associated with the risk of $\mathrm{BC}$ [33]. Zhang et al. reported that there were significantly lower BRCA1 mRNA levels among subjects with the rs799917 CC genotype than among those with the CT or TT genotypes in normal and cancerous esophageal tissues [25], which could explain why the risk of ESCC is lower among rs799917 T carriers.

Maia et al. performed an analysis of differential allelic expression (DAE) in BRCA1 rs799917 heterozygous individuals (CT individuals) [37]. They found that in fresh blood B cells, the T allele was expressed at a higher level than the $\mathrm{C}$ allele in some heterozygous individuals (44\%) and that the $\mathrm{C}$ allele was expressed at a higher level than the $\mathrm{T}$ allele in other heterozygous individuals (26\%). Furthermore, they also found that the $\mathrm{C}$ allele was consistently expressed at a higher level than the $\mathrm{T}$ allele in breast tissue in all heterozygous individuals. This suggests that in the complex cellular environment, the DAE of BRCA1 is not only influenced by miR-638 but also by other regulatory factors and that the regulatory factors for $B R C A 1$ rs799917 expression are different in fresh blood $B$ cells and breast tissue.

rs 1799950 is located in the region of exon 11 that binds Rad50, which is part of the DNA damage repair complex [38]. rs1799950 can be found in many families who are at a high-risk for prostate cancer [39]. Our meta-analysis revealed that rs 1799950 could decrease the breast cancer risk among Caucasian populations. rs 1799966 is located in the coding region of the $\mathrm{COOH}-$ terminal domain of BRCA1 (BRCT). The BRCT domain is an important signaling and protein-targeting motif in the DNA damage response system [40]. This suggests that rs1799966 may be important in the etiology of cancer. However, our results indicated that there was no observable association between rs1799966 and cancer risk, either in the overall or subgroup analysis. The presence of the rs16941 polymorphism has been reported to be significantly associated with the positive expression

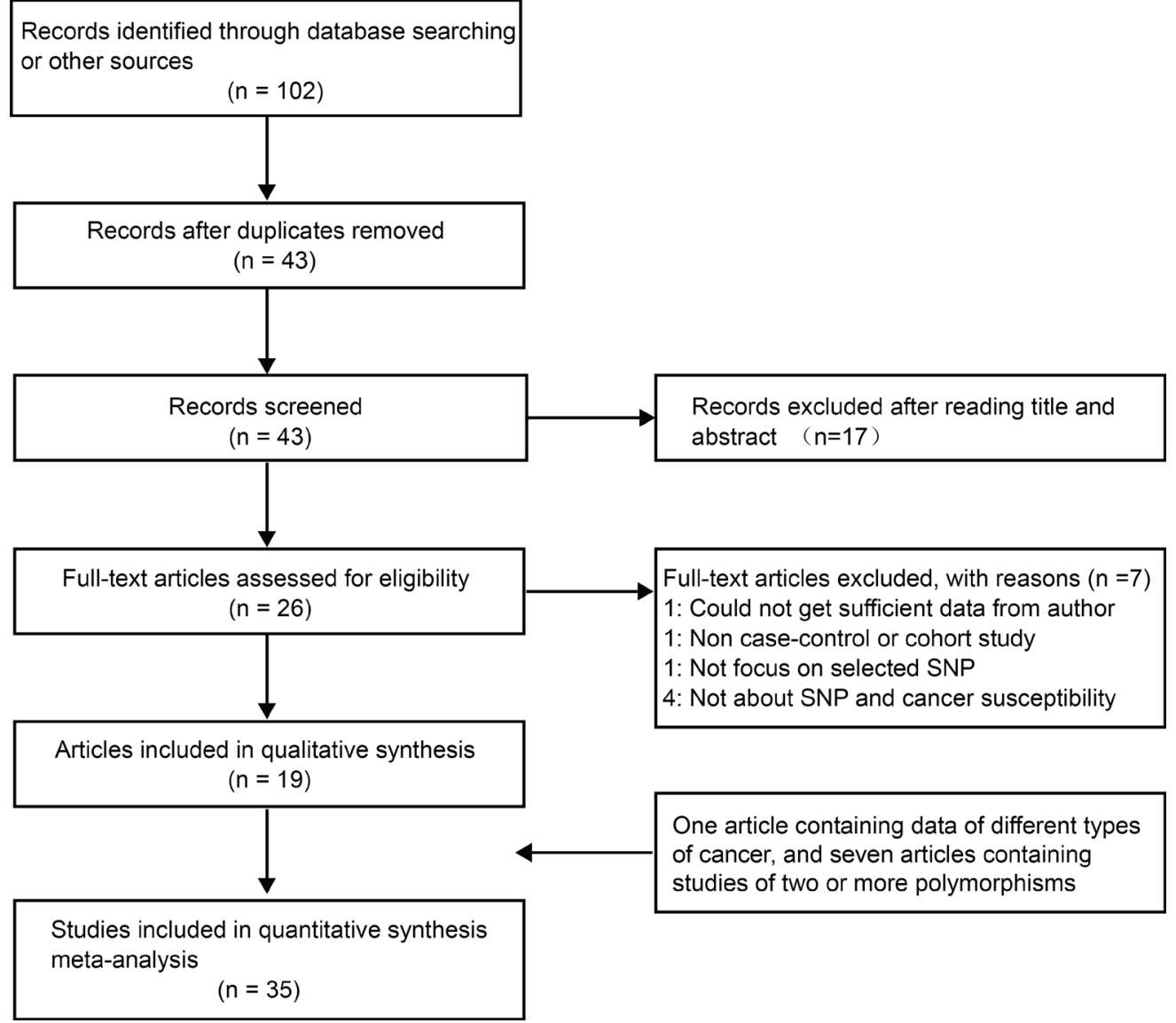

Figure 1: The flow diagram of included/excluded studies. 
of BRCA1 protein [41]; however, we found that rs 16941 could increase cancer risk among Caucasian populations. Therefore, we require more research to verify the results regarding rs16941 in the future.

There is nearly complete linkage disequilibrium between rs799917, rs1799966, and rs16941 in the most of the populations from the 1000 Genomes Project Phase 3 (Supplementary Figure 1). However, we ultimately chose to include these three SNPs instead of choosing only one because the studies on each SNP are very limited in number and the results for all the SNPs could complement one another and thus help to verify the conclusion. In our meta-analysis, we found that rs799917, rs1799966, and rs16941 were not associated with overall cancer risk. We suggest that among Asian populations rs799917 could decrease the risk of several types of cancer. However, for rs1799966 and rs16941, there are no studies about Asian populations included in the meta-analysis. We

A

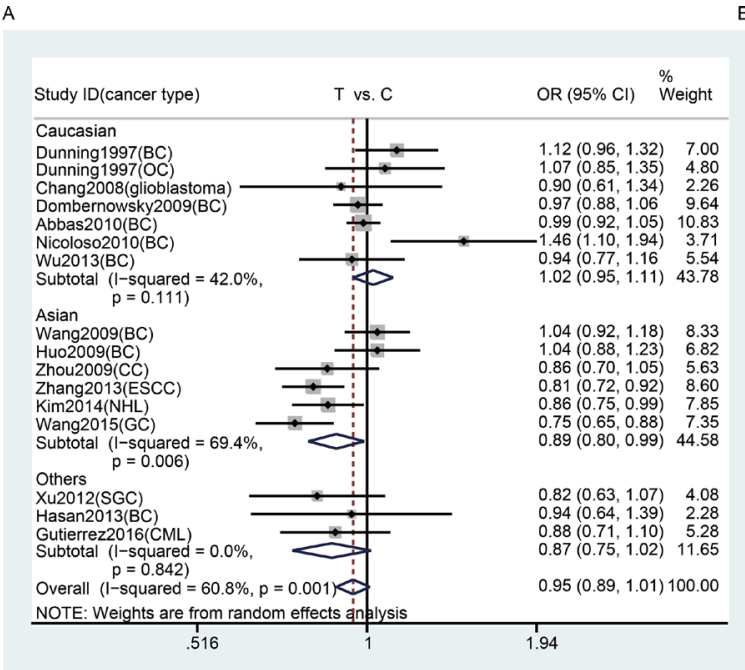

B
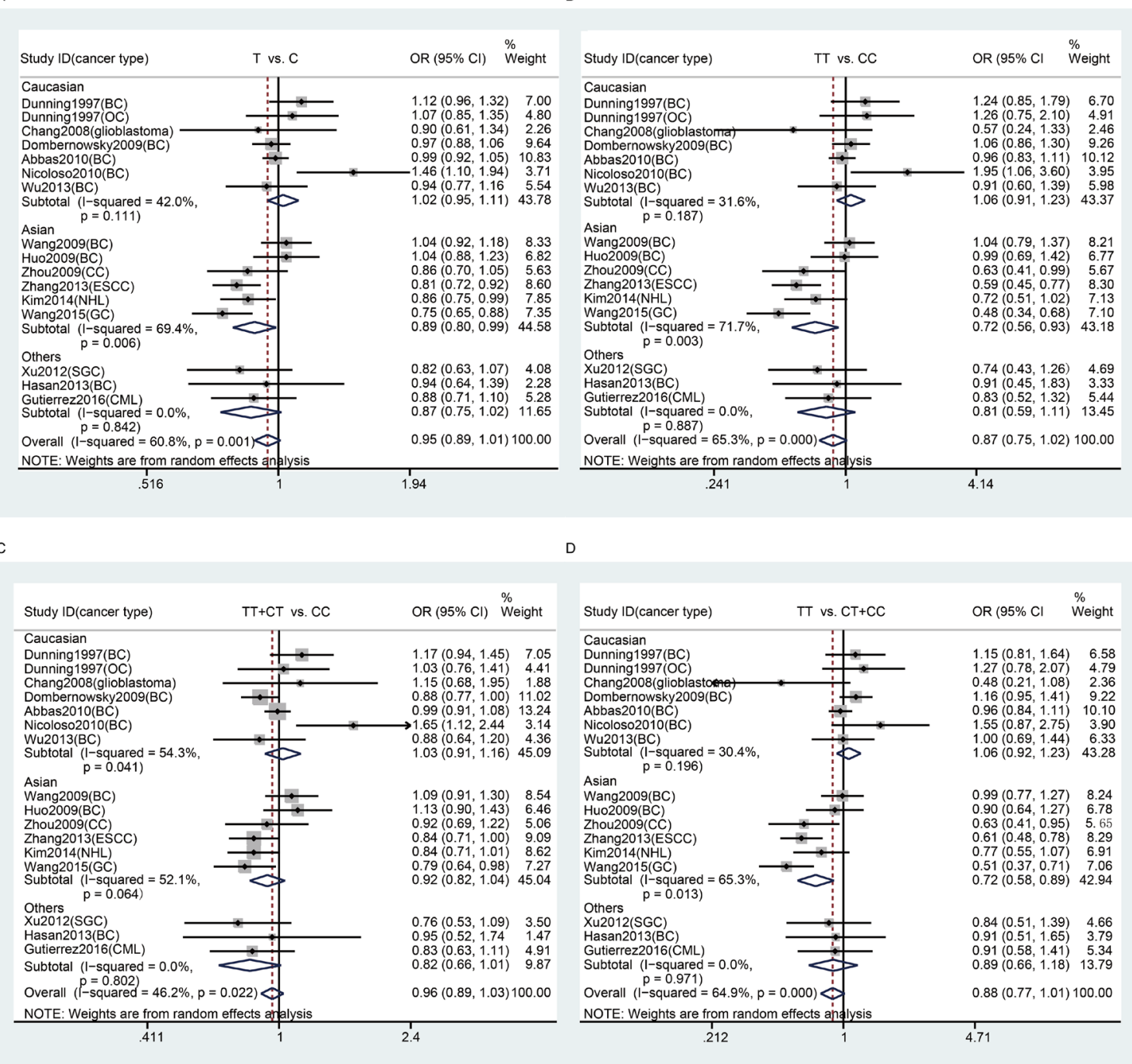

revealed that rs16941 could increase cancer risk among Caucasians but that rs799917 and rs1799966 could not. Taking into account the limited number of included studies and the fact that there was publication bias in the studies of rs 16941, we require more evidence to verify the conclusions in the future.

Our meta-analysis has certain limitations. First, we only included studies in the PubMed, Embase and Web of Science databases and those written in English. This means that some studies published in other databases and written in other languages may have been ignored. Second, the meta-analysis contained only a few types of cancer. Therefore, in the future, we will require more data regarding various types of cancer to arrive at a more comprehensive conclusion. Third, the results for rs799917 and rs16941 are unstable in some genetic models, and there is publication bias in the studies on rs16941. Thus, the conclusions drawn from the current

Figure 2: Stratification analyses by ethnicity between rs799917 polymorphism and cancer risk. (A) allele model; (B) homozygous model; (C) dominant model; (D) recessive model. The squares and horizontal lines correspond to the study specific OR and $95 \% \mathrm{CI}$. The area of the squares reflects the weight. The diamond represents the summary OR and $95 \%$ CI. The random-effects model was used. 
research should be interpreted with caution. Finally, because of the limited number of included studies, this meta-analysis did not consider gene-gene or geneenvironment interactions.

In conclusion, our meta-analysis suggests that rs799917 polymorphism could decrease the risk of cervical cancer, ESCC, gastric cancer, and NHL among Asian populations. We also found that rs1799950 could decrease breast cancer risk among Caucasian populations and that rs16941 could increase the overall cancer risk among Caucasian populations. Considering the limited number of cancer types in and the sample size of this meta-analysis, more studies including various types of cancer are needed to investigate the association between $B R C A 1$ polymorphisms and cancer risk in the future.

\section{MATERIALS AND METHODS}

\section{Search strategy}

We searched the PubMed, Embase, and Web of Science databases for relevant studies about BRCA1 polymorphisms and cancer risk that were published before July 31, 2017. The following keywords and

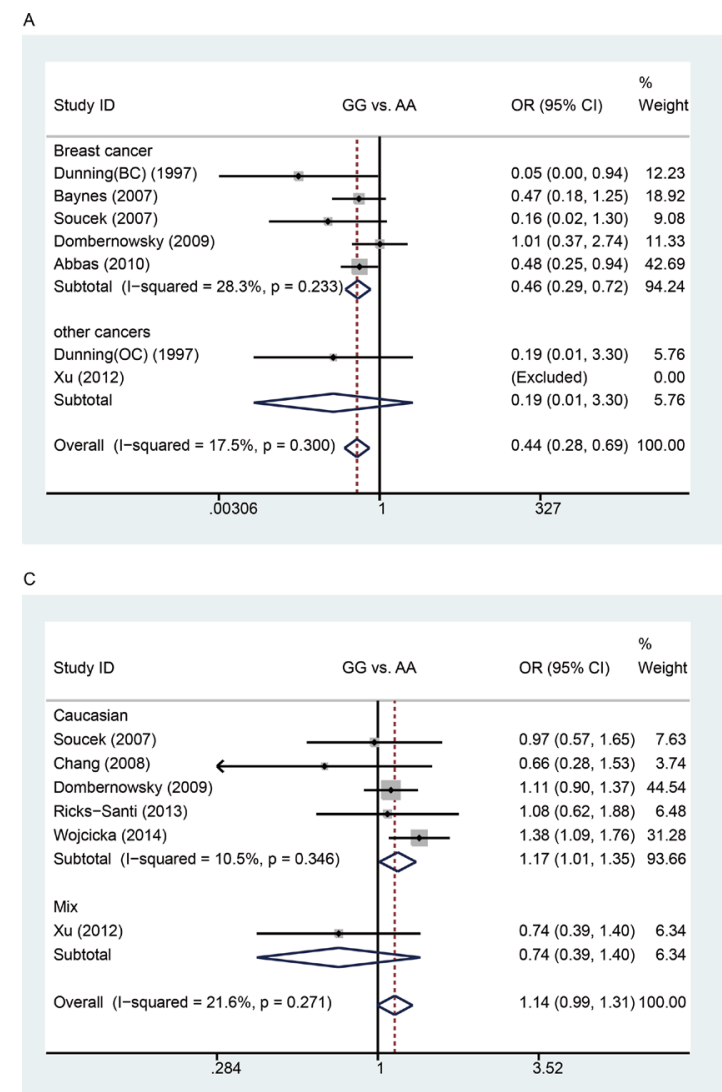

terms were used for the search: "BRCA1" or "Breast cancer associated gene 1"; "rs799917", "Pro871leu", “rs1799950", “Gln356Arg”, "rs1799966”, "Ser1613Gly”, "rs16941" or "Glu1038Gly"; "variant", "mutation", "polymorphism" or "SNP"; "cancer", "carcinoma", "neoplasm" or "tumor" and the combinations. Besides, the reference lists of identified studies were also screened carefully for potential articles.

\section{Inclusion and exclusion criteria}

All studies included in the meta-analysis had to meet the following criteria: 1) the study investigated the association between $B R C A 1$ polymorphisms and cancer risk; 2) the study was a case-control or cohort study; 3 ) the study was written in English; 4) the study contained detailed genotyping data. We excluded comments, editorials, reviews, meta-analyses, and studies lacking sufficient data.

\section{Data extraction}

Data were extracted from all eligible studies by two investigators (Gui-Ping $\mathrm{Xu}$ and Qing Zhao)

B

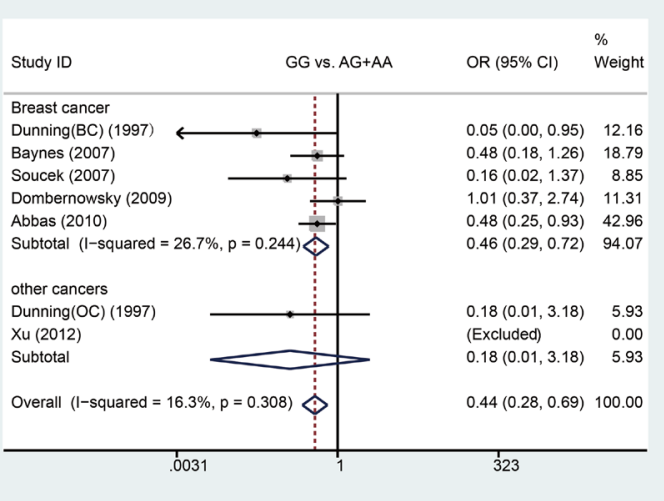

D

\begin{tabular}{|c|c|c|c|c|}
\hline \multirow{2}{*}{$\begin{array}{l}\text { Study ID } \\
\text { Caucasian }\end{array}$} & \multicolumn{2}{|c|}{$G G$ vs. $A G+A A$} & \multirow[t]{2}{*}{ OR $(95 \% \mathrm{Cl})$} & \multirow[t]{2}{*}{$\begin{array}{l}\% \\
\text { Weight }\end{array}$} \\
\hline & & & & \\
\hline Soucek (2007) & & & $0.91(0.55,1.50)$ & 8.15 \\
\hline Chang (2008) & & & $0.56(0.25,1.25)$ & 4.13 \\
\hline Dombernowsky (2009) & & & $1.18(0.96,1.44)$ & 43.38 \\
\hline Ricks-Santi (2013) & & & $1.01(0.59,1.72)$ & 6.76 \\
\hline Wojcicka (2014) & & $\rightarrow$ & $1.31(1.04,1.66)$ & 31.74 \\
\hline Subtotal $(I-$ squared $=27.1 \%$, & $p=0.241)$ & & $1.17(1.01,1.33)$ & 94.16 \\
\hline \multicolumn{5}{|l|}{ Mix } \\
\hline $\mathrm{Xu}(2012)$ & & & $0.83(0.45,1.55)$ & 5.84 \\
\hline Subtotal & 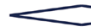 & 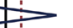 & $0.83(0.45,1.55)$ & 5.84 \\
\hline Overall $(I-$ squared $=23.6 \%, p$ & $p=0.257)$ & i & $1.14(1.00,1.31)$ & 100.00 \\
\hline .252 & & 1 & 3.97 & \\
\hline
\end{tabular}

Figure 3: Meta-analysis between rs1799950 and rs16941 polymorphisms and cancer risk. A and B: Stratification analyses by cancer type between rs1799950 polymorphism and cancer risk (A) homozygous model; (B) recessive model); C and D: Stratification analyses by ethnicity between rs16941 polymorphism and cancer risk (C) homozygous model; (D) recessive model). The squares and horizontal lines correspond to the study-specific OR and 95\% CI. The area of the squares reflects the weight. The diamond represents the summary OR and 95\% CI. The fixed-effects model was used. 
working independently. Disagreements were resolved via discussion. The following information was extracted: name of first author, year of publication, country, cancer type, ethnicity, genotyping methods, source of controls, genotype distributions of cases and controls, and HardyWeinberg equilibrium (HWE) for controls. One of the studies only provides the percentage of each genotype, the complete data were obtained from the author [32].

\section{Quality score}

The quality of the studies was independently evaluated by two reviewers (Ding Wang and Hua Zhou) based on a quality assessment scale (Supplementary Table 1). Any disagreement was resolved via discussion between the two reviewers. Total scores ranged from 0 (worst) to 15 (best) [42].
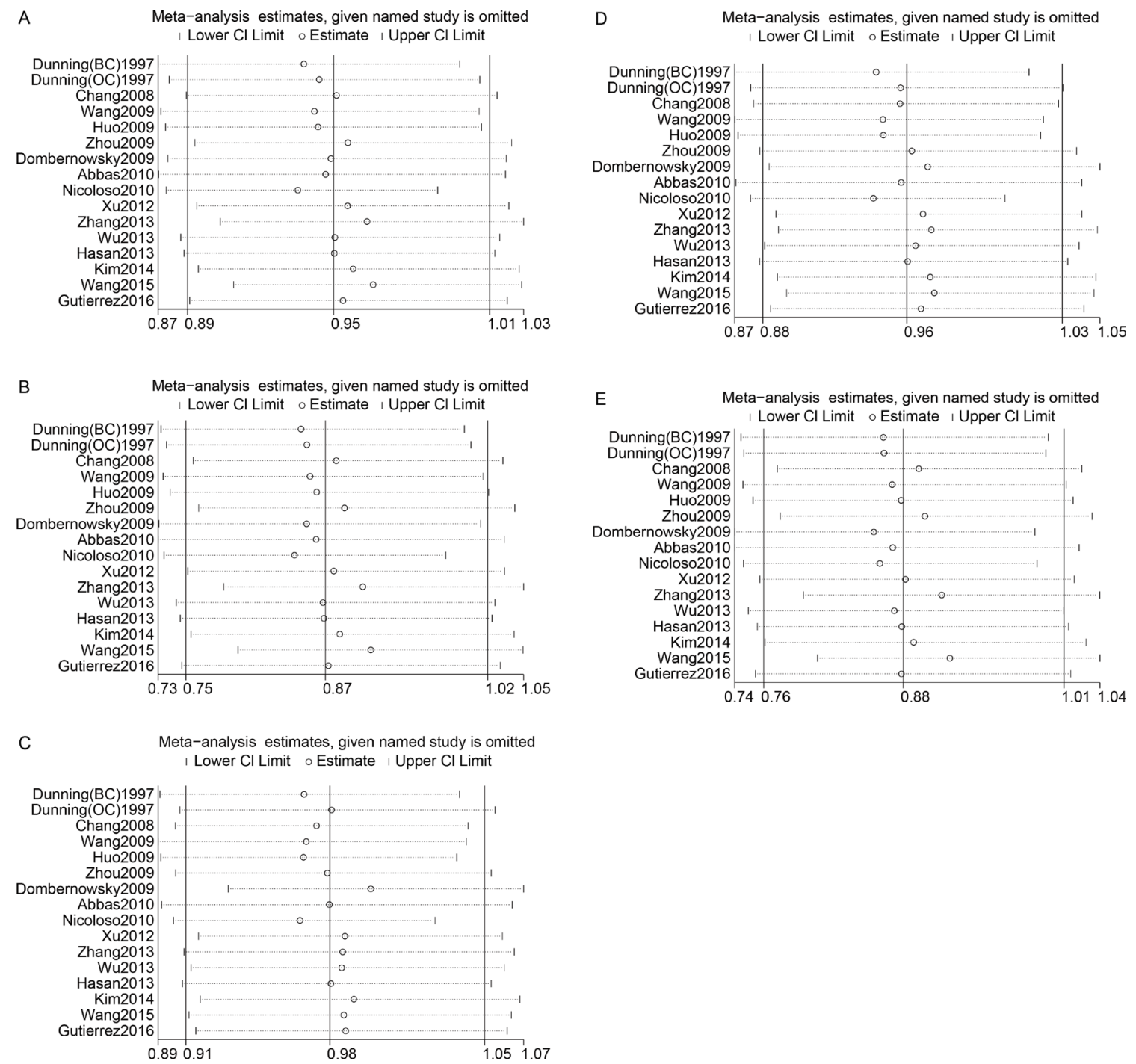

\section{Statistical analysis}

All statistical analyses were performed using the STATA software (Version 12.0; Stata Corporation, College Station, TX, USA). ORs with corresponding 95\% CIs were used to assess the strength of the association between $B R C A 1$ polymorphisms and cancer risk. The strength of the association was estimated in the allele genetic model (T vs. C), the homozygote model (TT vs. CC), the heterozygote model (CT vs. CC), the dominant model $(\mathrm{TT}+\mathrm{CT}$ vs. CC), and the recessive model (TT vs. CT + $\mathrm{CC})$. The significance of the pooled OR was determined with the Z-test, and $P$-values $<0.05$ were considered statistically significant. The heterogeneity among studies was evaluated using a chi-square-based $Q$ test and quantified by $\mathrm{I}^{2}[43]$. If the result of the heterogeneity test was $P>0.1$, which indicated that the heterogeneity among studies was not significant, ORs were pooled using the fixed-effects model (Mantel-Haenszel model) [44].

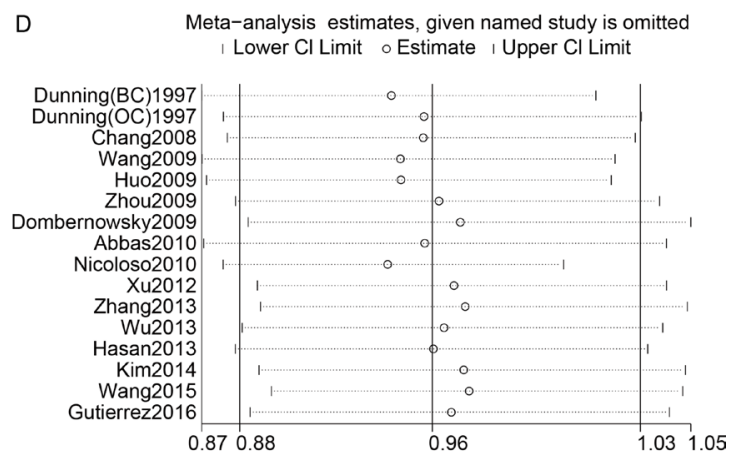


Otherwise, the random-effects model (DerSimonian and Laird model) was used [45]. Hardy-Weinberg equilibrium (HWE) in the control group for each study was examined using a chi-squared test and a $P$ value less than 0.05 was considered as significant disequilibrium. Stratified analyses were performed based on ethnicity, cancer type, control source, and quality score. Sensitivity analyses were performed to assess the stability of the results by omitting one single study each time. Begg's test and Egger's testwere performed to examine potential publication bias $[46,47]$. All $P$ values were two-sided, and $P<0.05$ was considered as statistically significant except for the $P$ value of heterogeneity.

\section{Abbreviations}

SNP, single nucleotide polymorphism; OR, odds ratio; $\mathrm{CI}$, confidence interval; $\mathrm{BC}$, breast cancer; $\mathrm{OC}$, ovarian cancer; SGC, Salivary Gland Carcinoma; ESCC, esophageal squamous cell carcinoma; NHL, NonHodgkin Lymphoma; PTC, Papillary Thyroid Carcinoma; CML, chronic myeloid leukemia; MAF, minor allele frequency; GWAS, genome-wide association study; HWE, Hardy-Weinberg equilibrium; DAE, differential allelic expression; ASOs: allele-specific oligonucleotides; PCR-PIRA: PCR primer introduced restriction analysis assay; PCR-RFLP: PCR restriction fragment length polymorphism; MALDI-TOF MS: matrix-assisted laser desorption/ionization time-of-flight mass spectrometry; PB: population-based; HB: hospital-based; FB: familybased.

\section{Author contributions}

Conceived and designed the experiments: GPX, LFW. Performed the experiments: QZ, DW, GPX, HZ. Analyzed the data: GPX, LFW, WYX, LJZ. Contributed reagents/materials/analysis tools: QZ, SZC. Wrote the paper: LFW, GPX.

\section{ACKNOWLEDGMENTS}

We thank Professor Emilio J Cordova for providing complete BRCA1 rs799917 genotype data for their study [32].

\section{CONFLICTS OF INTEREST}

The authors declare no competing financial interests.

\section{FUNDING}

This work was supported by grants from the National Natural Science Foundation of China (Grant No.
81601826), the Scientific and Technological Research Program of Chongqing Municipal Education Commission (Grant No.KJ1400202), and the Chongqing Health and Family Planning Commission Foundation (Grant No. 2013-2-046).

\section{REFERENCES}

1. Lichtenstein P, Holm NV, Verkasalo PK, Iliadou A, Kaprio J, Koskenvuo M, Pukkala E, Skytthe A, Hemminki K. Environmental and heritable factors in the causation of cancer-analyses of cohorts of twins from Sweden, Denmark, and Finland. N Engl J Med. 2000; 343:78-85. https://doi.org/10.1056/NEJM200007133430201.

2. Torre LA, Bray F, Siegel RL, Ferlay J, Lortet-Tieulent J, Jemal A. Global cancer statistics, 2012. CA Cancer J Clin. 2015; 65:87-108. https://doi.org/10.3322/caac.21262.

3. Köberle B, Koch B, Fischer BM, Hartwig A. Single nucleotide polymorphisms in DNA repair genes and putative cancer risk. Arch Toxicol. 2016; 90:2369-88. https://doi.org/10.1007/s00204-016-1771-2.

4. Hall JM, Lee MK, Newman B, Morrow JE, Anderson LA, Huey B, King MC. Linkage of early-onset familial breast cancer to chromosome 17q21. Science. 1990; 250:1684-89. https://doi.org/10.1126/science.2270482.

5. Miki Y, Swensen J, Shattuck-Eidens D, Futreal PA, Harshman K, Tavtigian S, Liu Q, Cochran C, Bennett LM, Ding W, Bell R, Rosenthal J, Hussey C, et al. A strong candidate for the breast and ovarian cancer susceptibility gene BRCA1. Science. 1994; 266:66-71. https://doi. org/10.1126/science.7545954.

6. Deng CX, Brodie SG. Roles of BRCA1 and its interacting proteins. BioEssays. 2000; 22:728-37. https://doi. org/10.1002/1521-1878(200008)22:8<728::AIDBIES6>3.0.CO;2-B.

7. Deng CX. BRCA1: cell cycle checkpoint, genetic instability, DNA damage response and cancer evolution. Nucleic Acids Res. 2006; 34:1416-26. https://doi.org/10.1093/nar/gk1010.

8. Deng CX. Tumor formation in Brcal conditional mutant mice. Environ Mol Mutagen. 2002; 39:171-77. https://doi. org/10.1002/em.10069.

9. Venkitaraman AR. Cancer susceptibility and the functions of BRCA1 and BRCA2. Cell. 2002; 108:171-82. https:// doi.org/10.1016/S0092-8674(02)00615-3.

10. Brodie SG, Deng CX. BRCA1-associated tumorigenesis: what have we learned from knockout mice? Trends Genet. 2001; 17:S18-22. https://doi.org/10.1016/S01689525(01)02451-9.

11. Thompson D, Easton DF, and Breast Cancer Linkage Consortium. Cancer Incidence in BRCA1 mutation carriers. J Natl Cancer Inst. 2002; 94:1358-65. https://doi. org/10.1093/jnci/94.18.1358.

12. Kadouri L, Hubert A, Rotenberg Y, Hamburger T, Sagi M, Nechushtan C, Abeliovich D, Peretz T. Cancer risks 
in carriers of the BRCA1/2 Ashkenazi founder mutations. J Med Genet. 2007; 44:467-71. https://doi.org/10.1136/ jmg.2006.048173.

13. Bougie O, Weberpals JI. Clinical considerations of BRCA1and BRCA2-mutation carriers: a review. Int J Surg Oncol. 2011; 2011:374012. https://doi.org/10.1155/2011/374012.

14. Dunning AM, Chiano M, Smith NR, Dearden J, Gore M, Oakes S, Wilson C, Stratton M, Peto J, Easton D, Clayton D, Ponder BA. Common BRCA1 variants and susceptibility to breast and ovarian cancer in the general population. Hum Mol Genet. 1997; 6:285-89. https://doi.org/10.1093/ hmg/6.2.285.

15. Baynes C, Healey CS, Pooley KA, Scollen S, Luben RN, Thompson DJ, Pharoah PD, Easton DF, Ponder BA, Dunning AM, and the SEARCH breast cancer study. Common variants in the ATM, BRCA1, BRCA2, CHEK2 and TP53 cancer susceptibility genes are unlikely to increase breast cancer risk. Breast Cancer Res. 2007; 9:R27. https://doi.org/10.1186/bcr1669.

16. Soucek P, Borovanova T, Pohlreich P, Kleibl Z, Novotny J. Role of single nucleotide polymorphisms and haplotypes in BRCA1 in breast cancer: Czech case-control study. Breast Cancer Res Treat. 2007; 103:219-24. https://doi. org/10.1007/s10549-006-9367-9.

17. Chang JS, Yeh RF, Wiencke JK, Wiemels JL, Smirnov I, Pico AR, Tihan T, Patoka J, Miike R, Sison JD, Rice T, Wrensch MR. Pathway analysis of single-nucleotide polymorphisms potentially associated with glioblastoma multiforme susceptibility using random forests. Cancer Epidemiol Biomarkers Prev. 2008; 17:1368-73. https://doi. org/10.1158/1055-9965.EPI-07-2830.

18. Wang Z, Xu Y, Tang J, Ma H, Qin J, Lu C, Wang X, Hu Z, Wang X, Shen H. A polymorphism in Werner syndrome gene is associated with breast cancer susceptibility in Chinese women. Breast Cancer Res Treat. 2009; 118:16975. https://doi.org/10.1007/s10549-009-0327-z.

19. Huo X, Lu C, Huang X, Hu Z, Jin G, Ma H, Wang X, Qin J, Wang X, Shen H, Tang J. Polymorphisms in BRCA1, BRCA1-interacting genes and susceptibility of breast cancer in Chinese women. J Cancer Res Clin Oncol. 2009; 135:1569-75. https://doi.org/10.1007/s00432-009-0604-6.

20. Zhou X, Han S, Wang S, Chen X, Dong J, Shi X, Xia Y, Wang X, Hu Z, Shen H. Polymorphisms in HPV E6/ E7 protein interacted genes and risk of cervical cancer in Chinese women: a case-control analysis. Gynecol Oncol. 2009; 114:327-31. https://doi.org/10.1016/j. ygyno.2009.05.011.

21. Dombernowsky SL, Weischer M, Freiberg JJ, Bojesen SE, Tybjaerg-Hansen A, Nordestgaard BG. Missense polymorphisms in BRCA1 and BRCA2 and risk of breast and ovarian cancer. Cancer Epidemiol Biomarkers Prev. 2009; 18:2339-42. https://doi.org/10.1158/1055-9965.EPI09-0447.

22. Abbas S, Beckmann L, Changclaude J, Hein R, Kropp S, Parthimos M, Dünnebier T, Hamann U, Brors B, Eils R,
Zapatka M, Brauch H, Justenhoven C, et al, and MARIEGENICA Consortium on Genetic Susceptibility for Menopausal Hormone Therapy Related Breast Cancer Risk. Polymorphisms in the BRCA1 and ABCB1 genes modulate menopausal hormone therapy associated breast cancer risk in postmenopausal women. Breast Cancer Res Treat. 2010; 120:727-36. https://doi.org/10.1007/s10549-009-0489-8.

23. Nicoloso MS, Sun H, Spizzo R, Kim H, Wickramasinghe P, Shimizu M, Wojcik SE, Ferdin J, Kunej T, Xiao L, Manoukian S, Secreto G, Ravagnani F, et al. Singlenucleotide polymorphisms inside microRNA target sites influence tumor susceptibility. Cancer Res. 2010; 70:278998. https://doi.org/10.1158/0008-5472.CAN-09-3541.

24. Xu L, Doan PC, Wei Q, Li G, Sturgis EM. Functional single-nucleotide polymorphisms in the BRCA1 gene and risk of salivary gland carcinoma. Oral Oncol. 2012; 48:84247. https://doi.org/10.1016/j.oraloncology.2012.03.012.

25. Zhang X, Wei J, Zhou L, Zhou C, Shi J, Yuan Q, Yang $\mathrm{M}$, Lin D. A functional BRCA1 coding sequence genetic variant contributes to risk of esophageal squamous cell carcinoma. Carcinogenesis. 2013; 34:2309-13. https://doi. org/10.1093/carcin/bgt213.

26. Ricks-Santi LJ, Nie J, Marian C, Ochs-Balcom HM, Trevisan M, Edge SB, Kanaan Y, Freudenheim JL, Shields PG. BRCA1 polymorphisms and breast cancer epidemiology in the Western New York exposures and breast cancer (WEB) study. Genet Epidemiol. 2013; 37:504-11. https://doi.org/10.1002/gepi.21730.

27. Wu HC, Delgado-Cruzata L, Machella N, Wang Q, Santella RM, Terry MB. DNA double-strand break repair genotype and phenotype and breast cancer risk within sisters from the New York site of the Breast Cancer Family Registry (BCFR). Cancer Causes Control. 2013; 24:2157-68. https:// doi.org/10.1007/s10552-013-0292-z.

28. Hasan TN, Shafi G, Syed NA, Alsaif MA, Alsaif AA, Alshatwi AA. Lack of association of BRCA1 and BRCA2 variants with breast cancer in an ethnic population of Saudi Arabia, an emerging high-risk area. Asian Pac J Cancer Prev. 2013; 14:5671-4. https://doi.org/10.7314/ APJCP.2013.14.10.5671.

29. Kim HN, Kim NY, Yu L, Kim YK, Lee IK, Yang DH, Lee JJ, Shin MH, Park KS, Choi JS, Kim HJ. Polymorphisms in DNA repair genes and MDR1 and the risk for non-Hodgkin lymphoma. Int J Mol Sci. 2014; 15:6703-16. https://doi. org/10.3390/ijms15046703.

30. Wójcicka A, Czetwertyńska M, Świerniak M, Długosińska J, Maciąg M, Czajka A, Dymecka K, Kubiak A, Kot A, Płoski R, de la Chapelle A, Jażdżewski K. Variants in the ATM-CHEK2-BRCA1 axis determine genetic predisposition and clinical presentation of papillary thyroid carcinoma. Genes Chromosomes Cancer. 2014; 53:516-23. https://doi.org/10.1002/gcc.22162.

31. Wang K, Xu L, Pan L, Xu K, Li G. The functional BRCA1 rs799917 genetic polymorphism is associated with gastric cancer risk in a Chinese Han population. Tumour Biol. 
2015; 36:393-97. https://doi.org/10.1007/s13277-0142655-9.

32. Gutiérrez-Malacatt H, Ayala-Sanchez M, Aquino-Ortega $\mathrm{X}$, Dominguez-Rodriguez J, Martinez-Tovar A, OlarteCarrillo I, Martinez-Hernandez A, Cecilia CC, Orozco L, Cordova EJ. C CC, Orozco L, Cordova EJ. The rs61764370 functional variant in the KRAS oncogene is associated with chronic myeloid leukemia risk in women. Asian Pac J Cancer Prev. 2016; 17:2265-70. https://doi.org/10.7314/ APJCP.2016.17.4.2265.

33. Qin TT, Chen T, Zhang Q, Du HN, Shu YQ, Luo K, Zhu LJ. Association between BRCA1 rs799917 polymorphism and breast cancer risk: A meta-analysis of 19,878 subjects. Biomed Pharmacother. 2014; 68:905-10. https://doi. org/10.1016/j.biopha.2014.08.006.

34. John EM, Miron A, Gong G, Phipps AI, Felberg A, Li FP, West DW, Whittemore AS. Prevalence of pathogenic BRCA1 mutation carriers in 5 US racial/ethnic groups. JAMA. 2007; 298:2869-76. https://doi.org/10.1001/ jama.298.24.2869.

35. Li WQ, Hu N, Hyland PL, Gao Y, Wang ZM, Yu K, Su H, Wang CY, Wang LM, Chanock SJ, Burdett L, Ding T, Qiao $\mathrm{YL}$, et al. Genetic variants in DNA repair pathway genes and risk of esophageal squamous cell carcinoma and gastric adenocarcinoma in a Chinese population. Carcinogenesis. 2013; 34:1536-42. https://doi.org/10.1093/carcin/bgt094.

36. Mullany LE, Wolff RK, Herrick JS, Buas MF, Slattery ML. SNP Regulation of microRNA expression and subsequent colon cancer risk. PLoS One. 2015; 10:e0143894. https:// doi.org/10.1371/journal.pone.0143894.

37. Maia AT, Spiteri I, Lee AJ, O'Reilly M, Jones L, Caldas $\mathrm{C}$, Ponder BA. Extent of differential allelic expression of candidate breast cancer genes is similar in blood and breast. Breast Cancer Res. 2009; 11:R88. https://doi.org/10.1186/ bcr2458.

38. Douglas JA, Levin AM, Zuhlke KA, Ray AM, Johnson GR, Lange EM, Wood DP, Cooney KA. Common variation in the BRCA1 gene and prostate cancer risk. Cancer Epidemiol Biomarkers Prev. 2007; 16:1510-16. https://doi. org/10.1158/1055-9965.EPI-07-0137.
39. Zuhlke KA, Madeoy JJ, Beebe-Dimmer J, White KA, Griffin A, Lange EM, Gruber SB, Ostrander EA, Cooney KA. Truncating BRCA1 mutations are uncommon in a cohort of hereditary prostate cancer families with evidence of linkage to 17q markers. Clin Cancer Res. 2004; 10:597580. https://doi.org/10.1158/1078-0432.CCR-04-0554.

40. Sheng ZZ, Zhao YQ, Huang JF. Functional evolution of BRCT domains from binding DNA to protein. Evol Bioinform Online. 2011; 7:87-97. https://doi.org/10.4137/ EBO.S7084.

41. Mangia A, Chiriatti A, Tommasi S, Menolascina F, Petroni S, Zito FA, Simone G, Schittulli F, Paradiso A. BRCA1 expression and molecular alterations in familial breast cancer. Histol Histopathol. 2009; 24:69-76. https://doi. org/10.14670/HH-24.69.

42. Tian X, Dai S, Sun J, Jiang S, Jiang Y. Association between TP53 Arg72Pro polymorphism and leukemia risk: a metaanalysis of 14 case-control studies. Sci Rep. 2016; 6:24097. https://doi.org/10.1038/srep24097.

43. Higgins JP, Thompson SG. Quantifying heterogeneity in a meta-analysis. Stat Med. 2002; 21:1539-58. https://doi. org/10.1002/sim.1186.

44. Mantel N, Haenszel W. Statistical aspects of the analysis of data from retrospective studies of disease. J Natl Cancer Inst. $1959 ; 22: 719-48$.

45. DerSimonian R, Laird N. Meta-analysis in clinical trials. Control Clin Trials. 1986; 7:177-88. https://doi. org/10.1016/0197-2456(86)90046-2.

46. Begg CB, Mazumdar M. Operating characteristics of a rank correlation test for publication bias. Biometrics. 1994; 50:1088-101. https://doi.org/10.2307/2533446.

47. Egger M, Smith GD, Schneider M, Minder C. Bias in metaanalysis detected by a simple, graphical test. BMJ. 1997; 315:629-34. https://doi.org/10.1136/bmj.315.7109.629. 